\title{
Monitoring $\mathrm{CO}_{2}$ Migration using Time-lapse Seismic Data
}

\author{
Brian J. Evans \\ Department of Exploration Geophysics, Curtin University of Technology, Perth, Australia and \\ Australian Petroleum Cooperative Research Centre Geodisc Program
}

\begin{abstract}
Time lapse seismic data provides an ability to track the progress of migrating $\mathrm{CO}_{2}$ during the injection process. When supercritical $\mathrm{CO}_{2}$ is injected into a formation, the formation's structure, seal, reservoir mineralisation, temperature and pressure dictates the fluid's migration pattern, and its state-of-phase.

Understanding the basic rock physics and interaction of $\mathrm{CO}_{2}$ with the associated PT relationships, mineralisation and seal status is a prerequisite for seismic monitoring, geophysically tracking the $\mathrm{CO}_{2}$ as it moves. This paper uses the conditions of Sleipner in the North Sea. As with any chemical mix, the rock matrix may be change state as a function of the presence of $\mathrm{CO}_{2}$. The result is a dynamic process requiring a seismic methodology for tracking $\mathrm{CO}_{2}$ migration within any reservoir. Consequently while $3 \mathrm{D}$ seismic is widely regarded as the preferred but expensive tool, other tools are emerging which may be more cost effective, but a knowledge of reservoir conditions is still a prerequisite to determining the optimum tool.
\end{abstract}

\section{Introduction}

At the Convention of Climate Change in Kyoto (1997), member nations agreed to reduce greenhouse gas emissions 5\% below 1990 levels, by 2012. One solution is long-term storage of $\mathrm{CO}_{2}$ within underground reservoirs. However, if it is to be stored safely and securely, a rigorous monitoring program is required to observe its movement during the injection process. Time-lapse 3-D (TL3-D) seismic methods have been developed by the petroleum industry to monitor the movement of hydrocarbon fluids within producing reservoirs to improve overall hydrocarbon recovery rates. This technology is expected to form the foundation for most monitoring programs involving $\mathrm{CO} 2$ storage in the future.

However, TL3-D may not be necessary in some geological structures, or where temperature and pressures may be variable, where time-lapse 2D may be adequate, or where cross-well tomography may be more cost-effective. Thus, a decision-making approach of which method to use is needed before decisions can be taken of the optimum monitoring approach.

This paper presents a methodology for determining the long-term storage of $\mathrm{CO}_{2}$, to allow a comprehensive monitoring program during the injection process.

\section{General Approach}

The commencing approach is to establish a rock physics model of the reservoir to be used. Injection of $\mathrm{CO}_{2}$ into sandstones saturated with brine will cause changes within the reservoir over time. For example, if supercritical $\mathrm{CO}_{2}$ is injected into a reservoir at $30^{\circ} \mathrm{C}$ and at a relatively low 
pressure in the order of $5 \mathrm{MPa}$, it is likely that it will form a gas bubble soon after injection. Alternatively, if the same conditions apply but the reservoir is at $15 \mathrm{MPa}$, it may remain in a supercritical state. By forward modelling the response to a rock matrix, estimates can be obtained of the changes in seismic velocity and amplitude associated with matrix changes. This will then determine whether a particular reservoir will respond better to compressional (P-) or shear ( $\mathrm{S}-$ ) waves.

As the $\mathrm{CO}_{2}$ moves through its life-cycle, it will change state-of-phase as a function of the reservoir mineralisation, pressure and temperature. For example, free $\mathrm{CO}_{2}$ will displace brine and migrate upward, passing through interface discontinuities. If the brine is free to move laterally away such as in an unconsolidated sand reservoir, little pore-pressure builds and it may be that the $\mathrm{CO}_{2}$ migration becomes a function of absorption and seepage. Alternatively, $\mathbf{f}$ there is pore-pressure build-up, the migrating fluids may open pre-existing fractures in the matrix.

The seal will finally act as a structural trap (Johnson et al., 2001) of the vertically migrating $\mathrm{CO}_{2}$. After some time, it will begin to dissolve into the brine, which may cause it to descend again as brine becomes heavier when satur ated with $\mathrm{CO}_{2}$. This dissolution into the brine releases hydrogen and bicarbonate ions (Johnson et al., 2001), reducing the $\mathrm{pH}$ of the brine and the acidic conditions will now dissolve minerals within the matrix, causing a slight porosity increase thereby changing the seismic properties of the matix.

The breakdown of these minerals may release metallic cations such as calcite and dolomite, a process known as mineral trapping. Hence, the continued development of carbonates over the long term reduces porosity and permeability, while increasing density and likely cementing rock grains together.

The seismic response to small changes in $\mathrm{CO}_{2}$ from the liquid to gas phase is known to be large. Changes caused by porosity variations will also appear in any time lapse seismic data, and increased density with cementation will also be expected to produce a positive seismic response. Thus, modelling these responses becomes necessary before seismic monitoring commences.

\section{Rock Physics modelling}

A favoured reservoir condition for $\mathrm{CO}_{2}$ is that of unconsolidated sands aquifer reservoirs, such as the Utsira sands at the Sleipner injection site in the North Sea. This reservoir is situated within the Norwegian sector and is located between $600 \mathrm{~m}$ and $1100 \mathrm{~m}$ depth below the sea-bed with a porosity of around 38\%. A numerical model is initially required which predicts the effect of fluid and mineral substitution, as well as changes in porosity, on the seismic properties of this reservoir. By comparing the $\mathrm{P}$ - and S-wave velocities with those measured within the sand, an appropriate rock physics model can be developed.

Assuming the formation is isotropic and consisting of a composite porous rock saturated with a mixture of immiscible fluids, the velocities are dependent on the bulk modulus, shear modulus and density. The effective grain moduli can be calculated as a function of the mineral composition and porosity, located within the Hashin-Shtrikman (1963) effective elastic moduli bounds. For the Utsira, these bounds are small and provide an approximation of the effective grain moduli (Mavko et al., 1998). 
The Gassmann equations provide a background for the estimation of frame moduli, with the theoretical Walton (1987) variation being the closest for determining velocities which are comparable with those observed in the Utsira. The Utsira mineralogy was input to this model so that the effective grain elastic moduli could be calculated along with the changes in seismic properties of an Utsira-like reservoir during long-term storage. Using the estimated porosity and effective pressure result for such a reservoir, the velocity and density profile (Figure 1) should provide a good match with the measured sonic velocities.

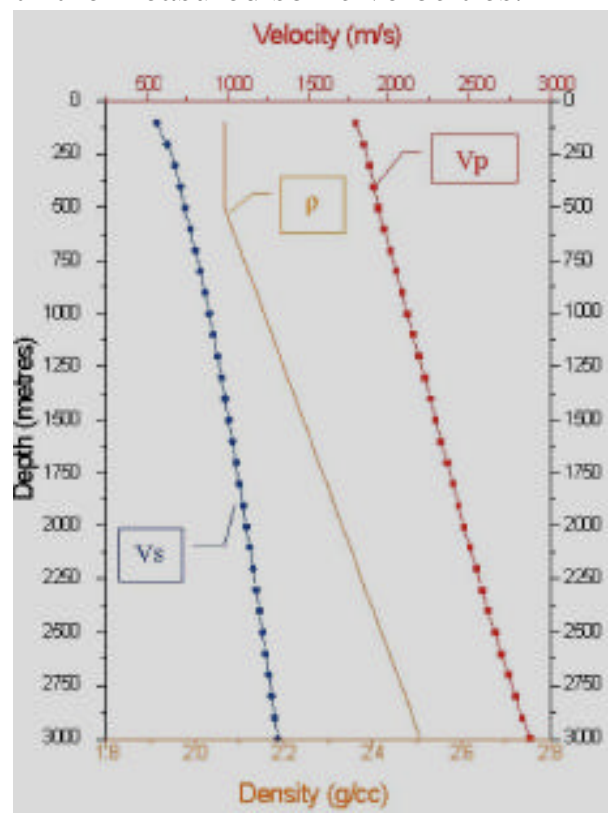

Figure 1. Using porosity and effective pressure gradients of an Utsira-like reservoir, modelled velocity and density gradients can be developed.

Due to the multitude of variations in reservoir conditions along with a need to understand the changes in velocity, it is now possible to develop relationship figures for depth versus percent $\mathrm{CO}_{2}$ saturation, reservoir temperature and $\mathrm{P}$-wave velocity. In Figure 2(a), the P-wave velocity is plotted as an iso-velocity change contour for three fixed P-wave values of $-650 \mathrm{~m} / \mathrm{s},-450 \mathrm{~m} / \mathrm{s}$ and $-250 \mathrm{~m} / \mathrm{s}$. The negative denotes a reduction in $\mathrm{P}$-wave values due to the injection of supercritical $\mathrm{CO}_{2}$ at a modelled temperature of $60^{\circ} \mathrm{C}$, and the location of the Utsira sands on this figure would be in the region as shown by the arrow (McKenna et al., 2003).

By comparison, the shear-wave equivalent is shown in Figure 2(b) where it is clear that the minimal increase in S-wave velocity suggests that Swaves would not be a good indicator of saturation changes, which is of particular importance in monitoring $\mathrm{CO}_{2}$ movement, particularly in reservoirs such as the Utsira which have low pressure and high temperature regimes. 


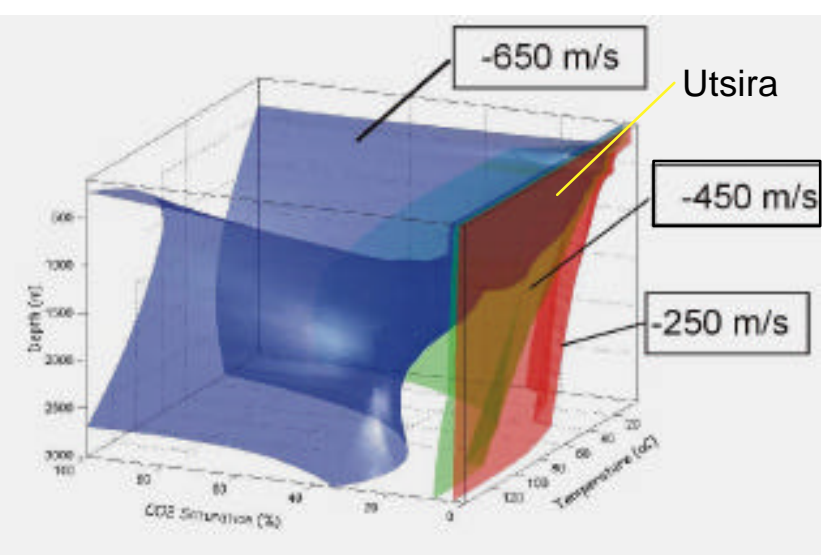

(a)

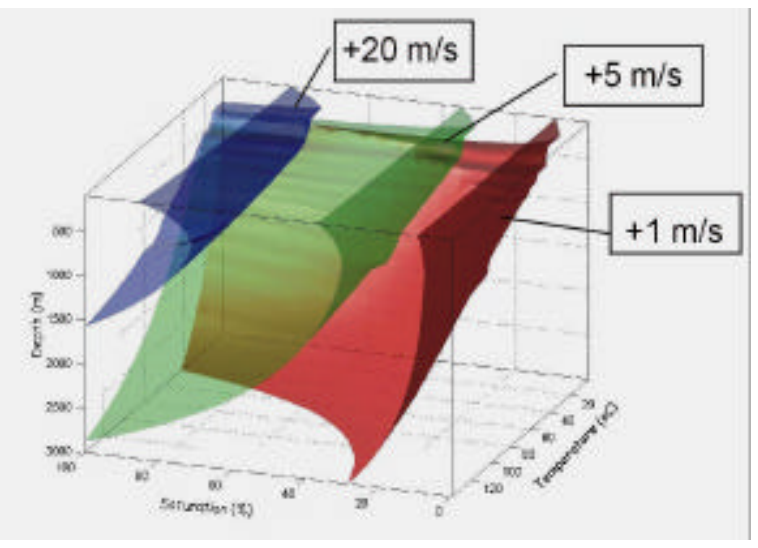

(b)

Figure 2. Changes in (a) $\mathrm{P}$-wave and (b) $\mathrm{S}$-wave velocity as a function of $\mathrm{CO}_{2}$ displacement of brine within an unconsolidated sand reservoir.

\section{Seismic sensing}

Because $\mathrm{CO}_{2}$ is more compressible than brine, the displacement of brine causes the P-wave velocity to decrease. At low pressure and high temperature, a vapour-phase $\mathrm{CO}_{2}$ can cause a $\mathrm{P}$-wave reduction greater than $650 \mathrm{~m} / \mathrm{s}$ while increased pressure maintains $\mathrm{CO}_{2}$ in a liquid phase causing reduction in velocity variations and hence, lack of seismic monitoring efficiency.

The dissolution into the brine may cause susceptible materials to dissolve, increasing porosity, thereby allowing TL3-D seismic differencing to work. The precipitation of carbonates mentioned earlier, alters the mineralogy of the reservoir. The net product is an increase in carbonate cementation between grains, resulting in an increase in the elastic moduli, velocity and density.

It is accepted that amplitudes as well as seismic frequencies reduce as body waves pass through a gas-saturated rock matrix. $\mathrm{CO}_{2}$ can be treated equally in terms of the ability to use TL3-D data to observe changes in amplitudes and frequencies due to changes in $\mathrm{CO}_{2}$ phase. It is also well accepted that they respond to changes in rock density and strength, by increased reflectivity and a reduction in frequency attenuation.

In more confined reservoirs where the main trapping mechanism is the seal, it becomes necessary to consider the mineralogy of the seal. For example, if there were the required elements within the seal matrix, it could be possible that the seal could either be breached due to seal dissolution whereas the seal could alternatively experience the development of carbonates, and thus hinder the flow of $\mathrm{CO}_{2}$. In a pressure regime which allows an increase in pore pressure, the seal may experience a vertical buoyancy pressure applied from beneath, resulting in a change in seal elastic parameters. Hence, seismic anisotropy studies become of extreme value in determining the state of the seal, before and during the $\mathrm{CO}_{2}$ injection phase of operations.

Seismic amplitudes, P-wave velocity, S-wave velocity and their associated Vp/Vs ratios, as well as frequency can all be used for tracking $\mathrm{CO}_{2}$ phase changes within the reservoir. However, the seal is also of extreme importance, since there is no point in mapping pre-injection reservoir size and ability to absorb the $\mathrm{CO}_{2}$ levels if the seal is compromised. 


\section{Monitoring methodology}

There is a preference to store $\mathrm{CO}_{2}$ in reservoirs which have a large volume, have a high porosity and permeability, have relatively low pressure and temperature, and are adequately sealed. Unconsolidated sand reservoirs provide such a scenario where the injection of $\mathrm{CO}_{2}$ into the formation waters causes little increase in reservoir pressure allowing the displacement of water to be a relatively easy event.

In order to determine the optimum location for storing $\mathrm{CO}_{2}$, studies commence with a site specific economic and environmental review. Assuming that the $\mathrm{CO}_{2}$ must be stripped from the host medium, the optimum location requires an economic and environmentally acceptable site. A reservoir volumetric assessment must be performed which includes mapping the reservoir both in volume and orientation, since it is to be expected that the fluids will move during injection and over time. To maintaining minimum monitoring costs, it would be ideal to maintain the injected fluids at one location, but this depends upon the reservoir characteristics and seal geometry.

When a structural geometry is expected of anticlinal form, cross-well P-wave tomography may be one low -cost monitoring approach, where migrating fluids change in depth over time, rather than spread laterally, which is the long-term expectation of fluids in a structure-free (flat) reservoir. In an anticlinal geometry, faulting and fracturing can also be expected. However, it may be that fracturing within reservoir sands pre-injection is not evident on the seismic data, with or without anisotropic studies. This is also true of an anticlinal seal, where fracturing can be most unwelcome. Thus, injection into structural traps is not high on the priority list whereas large, horizontal reservoirs are preferred.

A flatreservoir allows the lateral spread of fluids. Where pre-injection seismic may not show fractures present (which is very important for pre-injection reservoir simulation studies), it may be that thin shale compartments or boundaries may not be evident on pre-injection seismic data. These become quite evident after injection, as $\mathrm{CO}_{2}$ becomes trapped beneath them. In addition, any local build-up in $\mathrm{CO}_{2}$ can cause a local pressure increase, thereby opening previously closed fractures. In addition, the dissolution of rock matrix with resulting increase in porosity, with the subsequent increase in carbonate formation, causes changes in the rock fabric which can be detected through the use of areal 3-D seismic methods rather thanthe single bore-hole methods.

\subsection{Conventional TL3-D}

Conventional TL3-D methods are useful when comparing areal-lateral movement of $\mathrm{CO}_{2}$. A 3-D survey is initially recorded prior to the start of injection, which is then used for establishing reservoir conditions, such as structure, depth, reservoir extent, faulting and geological studies. The seal is of equal importance, with particular reference to its overall geometry and sealing capability. Anisotropy studies of the seal are a pre-requisite to any reservoir study since a fractured seal should be disregarded from the outset. Fortunately, the seismic response to changes in shale is often more pronounced than the response to changes in sands, so conventional 3D seismic is useful. For such studies as these, a reasonably long $(6 \mathrm{~km})$ streamer offset is preferred.

The main problem with using repeated 3-D surveys is that of cost. For example, the cost of a 
TL3-D survey can be in the order of $\$ 3$ million, and if the reservoir does not come through the fracture studies well, they must be repeated as soon as possible, perhaps every two years. In competent reservoirs, the industry is performing repeated surveys to be every five years. All the same, this is an expense which adds to the overall cost of sequestration operations.

\subsection{Continuous 3-D}

By comparison, evolving technologies are indicating that by placing continuous vibrating piezo-electric type sources with static receivers in the ground or sea-bed above the reservoir, then the continous time-lapse variations in fluid movement or phase changes can be tracked as they happen. This approach has been demonstrated in underground gas- storage reservoirs in France. 'SeisMovie', a trade-mark of CGG, uses differences in phase between continuous seismic signals to sense the changes as they happen. Once such a system is installed, the economics become little more than processing small amounts of data. This method offers a realistic approach to the future economic-fluid monitoring nexus.

\section{Conclusions}

The movement of $\mathrm{CO}_{2}$ through a reservoir sand is a dynamic process, and is dependent upon the reservoir rock properties, mineralisation, structure, porosity, permeability, saturation, temperature and pressure. The long-term storage is also dependent upon seal integrity and volume. Prior to any monitoring exercise, these properties must be fully understood so that after injection, changes in their state may be assessed. The result of the rock physics and reservoir/seal property study determines the form and extent of time-lapse survey required for monitoring its movement.

\section{Acknowledgements}

The author acknowledges the support of Kyoto University for the presentation of this paper and strong input from Jason McKenna and Boris Gurevich.

\section{References}

Hashin, Z and Shtrikman, S., 1963, A variational approach to the elastic behaviour of multiphase materials. In: Journal of the Mechanics and Physics of Solids, 11, 127-140.

Johnson, J.W., Nitao, J.J., Steefel, C.I. and Knauss, K.G., 2001, Reactive transport modelling of geologic $\mathrm{CO}_{2}$ sequestration in saline aquifers: the influence of intra-aquifer shales and the relative effectiveness of structural, solubility and mineral trapping during prograde and retrograde sequestration: $1^{\text {st }}$ National Conference on Carbon Sequestration, Washington.

Mavko, G., Mukerji, T., and Dvorkin, J., 1998, The Rock Physics Handbook - Tools For Seismic Analysis in Porous Media: Cambridge University Press.

McKenna, J. J., Gurevich, B., Urosevic, M., and Evans, B.J., 2003, Estimating buk and shear modulus for shallow saline a quifers undergoing $\mathrm{CO}_{2}$ injection: Paper accepted by SEGJ, Tokyo.

Walton, K., 1987, The effective elastic moduli of a random packing of spheres: Journal of the Mechanics and Physics of Solids, 35, 213-226. 